\title{
Dynamic modeling and control method of a new concept wing-disk aircraft
}

\author{
Da Hong ${ }^{1, *}$, and Jihong $\mathrm{Zhu}^{2}$ \\ ${ }^{1}$ Naval Research Academy, Beijing, China \\ ${ }^{2}$ School of Computer Science and Technology, Tsinghua University, Beijing, China
}

\begin{abstract}
The research aimed at a new layout wing-disk solar aircraft concept with several wings around the disc fuselage, select coordinates and variables adaptively for the new dynamic subject, and establish dynamic model, using blade element momentum theory and CFD value for correction. Design the flight control strategy and controller constraint relations, put forward the method of control allocation and manipulation, and use nonlinear dynamic inversion control method aimed at the transverse and longitudinal coupling, serious nonlinear characteristics, and adds integral element as a robust dynamic inverse control to deal with the poor performance of previous method, and simulate to validate the control design.
\end{abstract}

\section{Introduction}

Disk or saucer shaped aircraft is in form of culvert or rotor generally, with compact structure and high stiffness, is a significance reference for the design of solar aircraft. Ref [1] proposes a wing-disk solar-powered aircraft with several moderate aspect ratio wings arranged around the disk fuselage. The wings and fuselage can both provide lift while the overall rotation and forward flying. Propeller at the outer end of the wings is used to overcome the resisting moment. Height and position is controlled by changing the propeller thrust and rotary speed of the disc, and attitude is controlled by adjusting the aileron rudder deflection angle. Medium aspect ratio wings can provide better stiffness, the wing disc can also obtain larger laying area of solar photovoltaic component and load capacity, which is a new idea of layout for a solar powered aircraft.

Ref. [1] carry out overall design for the aircraft, put forward reasonable layout, size, weight and flight parameters included. The research proceeds dynamic modeling and control method, which is of great significance to flight mechanism research and the detail design of the new concept aircraft. The layout and flight mechanism of the wing-disk aircraft is shown in figure 1 .

\footnotetext{
*Corresponding author: honda198829@126.com
} 

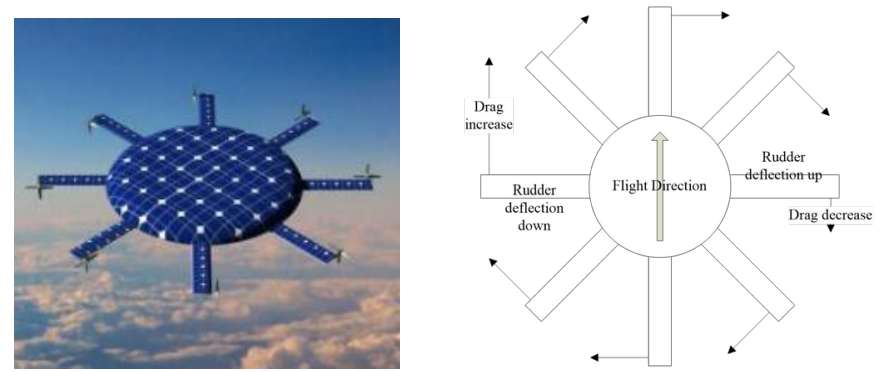

Fig. 1. The layout and flight mechanism of the wing-disk aircraft.

\section{Dynamic Modeling}

\subsection{Coordinate System}

It is necessary to select the appropriate coordinate system according to the characteristics of nearly axisymmetric and always rotating. Set $O$ is center of gravity, established coordinate systems of ground $\boldsymbol{O} \boldsymbol{X}_{d} \boldsymbol{Y}_{d} \boldsymbol{Z}_{\boldsymbol{d}}$, body $\boldsymbol{O \boldsymbol { X } _ { b }} \boldsymbol{Y}_{b} \boldsymbol{Z}_{\boldsymbol{b}}$ and track $\boldsymbol{O} \boldsymbol{X}_{h} \boldsymbol{Y}_{h} \boldsymbol{Z}_{h}$, and $\boldsymbol{O} \boldsymbol{X}_{d}$ is the initial heading, $\boldsymbol{O} \boldsymbol{X}_{\boldsymbol{b}}$ is along a wing (wing number $i=1$ ) and outward positive, $\boldsymbol{O}_{\boldsymbol{h}}$ is along the flight speed direction $\boldsymbol{V}, \boldsymbol{O Z}$ is generally downward positive, $\boldsymbol{O} \boldsymbol{Y}$ is determined by the right hand rule, set $\boldsymbol{L}$ is coordinate transformation matrix.

According to the definition of generalized Euler angles, use the Cardan angle definition method, set the conversion between the ground coordinate system to the body of the coordinate system is passed around the shaft $\boldsymbol{X}, \boldsymbol{Y}$ and $\boldsymbol{Z}$, and the rotating roll angle $\varphi$, pitch angle $\theta$ and rotation angle $\psi$. The rotate angle $\psi \in(0, \infty)$ comprises two parts of information, $\psi=J \cdot 2 \pi+K$ is a natural number, where $J$ reflects the number of the rotating, and increases during the flight, so $K \in(0,2 \pi)$. In addition, the inclination angle $\gamma$ and azimuth $\chi$ of the track are defined generally.

\subsection{Dynamics Equations}

\subsubsection{Expression in Vector}

Ignore the ailerons and propellers, the aircraft can be regarded as a rigid body, the dynamic model is expressed as Equation (1-2).

$$
\begin{aligned}
& m \frac{d \boldsymbol{V}}{d t}=\sum_{i=1}^{8} \boldsymbol{T}_{i}+\boldsymbol{G}+\boldsymbol{F} \\
& \boldsymbol{I} \cdot \frac{d \boldsymbol{\omega}}{d t}+\boldsymbol{\omega} \times(\boldsymbol{I} \cdot \boldsymbol{\omega})=\boldsymbol{M}_{R}+\boldsymbol{M}_{T}
\end{aligned}
$$

where $\boldsymbol{V}$ is the speed of the aircraft, $\boldsymbol{T}_{i}$ for the pull of power equipped with wing number $i$, $\boldsymbol{G}$ is gravity, $\boldsymbol{F}$ is aerodynamic force. $\boldsymbol{\omega}$ is the rotation speed in inertial system, $\boldsymbol{M}_{R}$ is moment of the aerodynamic force relative center of gravity, $\boldsymbol{M}_{T}$ is moment of the pull of power. $\boldsymbol{I}$ is matrix of inertia.

\subsubsection{Expression in Coordinate system}

According to the characteristics of the axisymmetric rotating motion, the movement of the center of mass in the ground coordinate system can be obtained as Equation 3. 


$$
\begin{aligned}
& m \frac{d v_{x}}{d t}=\sum_{i=1}^{8} T_{i d x}+F_{d x} \\
& m \frac{d v_{y}}{d t}=\sum_{i=1}^{8} T_{i d y}+F_{d y} \\
& m \frac{d v_{z}}{d t}=\sum_{i=1}^{8} T_{i d z}+m g+F_{d z}
\end{aligned}
$$

where $\boldsymbol{F}_{d x}, \boldsymbol{F}_{d y}, \boldsymbol{F}_{d z}$ are air force in ground coordinate system, $\boldsymbol{T}_{i d x}, \boldsymbol{T}_{i d y}, \boldsymbol{T}_{i d z}$ are pull force of number $i$ in ground coordinate system. It is necessary to take into account that the vehicle has characteristics of approximate axial symmetry, namely $\boldsymbol{I}_{x y}=\boldsymbol{I}_{y z}=\boldsymbol{I}_{x z}=0$ and $\boldsymbol{I}_{y}=\boldsymbol{I}_{x}$, the rotation can be expressed in coordinate system. The Equation (4) as:

$$
\begin{aligned}
& \dot{p}=\frac{1}{I_{x}}\left[M_{b x}-\left(I_{z}-I_{y}\right) q r\right] \\
& \dot{q}=\frac{1}{I_{y}}\left[M_{b y}-\left(I_{x}-I_{z}\right) p r\right] \\
& \dot{r}=\frac{1}{I_{z}}\left(M_{b z}+M_{T}\right)
\end{aligned}
$$

where $\boldsymbol{M}_{b x}, \boldsymbol{M}_{b y}, \boldsymbol{M}_{b z}$, are the moment of the force in the body coordinate system, $\boldsymbol{M}_{T}$ is the moment of the force of the power in body coordinate system.

\subsection{Model Integrating}

According to the flight characteristics of aircraft rotation, choose three speed $v_{x}, v_{y}, v_{z}$ in ground coordinate system and three angular velocity $p, q, r$ in body coordinate system to describe the vehicle instantaneous speed, with the roll angle $\phi$, pitch angle $\theta$ and rotation angle $\psi$, all together 9 Euler angles to describe the status of aircraft flight. The aerodynamic analysis involves speed in body coordinate system $u, v$ and $w$, velocity $V$ and flight path angle $x$ and $\gamma$ in conversion between coordinates, the above 6 can be regarded as intermediate variables, add 3 groups movement relationship appropriately. The Equation (5-7) are as follow:

$$
\begin{gathered}
{\left[\begin{array}{c}
p \\
q \\
r
\end{array}\right]=\boldsymbol{L}_{b d}\left[\begin{array}{c}
\dot{\phi} \\
0 \\
0
\end{array}\right]+\boldsymbol{L}_{b d}^{z}(\psi) \boldsymbol{L}_{b d}^{v}(\theta)\left[\begin{array}{c}
0 \\
\dot{\theta} \\
0
\end{array}\right]+\boldsymbol{L}_{b d}^{z}(\theta)\left[\begin{array}{c}
0 \\
0 \\
\dot{\psi}
\end{array}\right]} \\
{\left[\begin{array}{c}
v_{x} \\
v_{y} \\
v_{z}
\end{array}\right]=\boldsymbol{L}_{d h}\left[\begin{array}{c}
V \\
0 \\
0
\end{array}\right]} \\
{\left[\begin{array}{c}
u \\
v \\
w
\end{array}\right]=\boldsymbol{L}_{d h}\left[\begin{array}{c}
V \\
0 \\
0
\end{array}\right]}
\end{gathered}
$$

\subsection{Main Parameters}

\subsubsection{Aerodynamic Force}

Ref [1] use blade element method and CFD simulation to get aerodynamic performance, blade element method mainly aims at the part of wings, CFD is effective on the whole, but 
the description is not detail enough, only used in disc body part. Namely, use different method for the rotor and disc body parts (subscript $R, 0$ ).

The rotation of cruise flight is not too fast, use blade element method to carry out the aerodynamic analysis, and get lift $d L$, drag $d D$, and pitching moment $d m$ of a blade element $d x$, and the lift $d U$, drag $d Q$ and torque $d M$ in the body coordinate system with integral. Rotor aerodynamic forces in body coordinate system can be expressed as Equation (8).

$$
\begin{aligned}
F_{R b x} & =\int_{x=R}^{x=R+l} \sum_{i=1}^{8} \sin \frac{(i+3) \pi}{4} d Q_{i} \\
F_{R b y} & =\int_{x=R}^{x=R+l} \sum_{i=1}^{8} \sin \frac{(i+5) \pi}{4} d Q_{i} \\
F_{R b z} & =\int_{x=R}^{x=R+l} \sum_{i=1}^{8} d U_{i}
\end{aligned}
$$

where $R$ is the radius of the fuselage, $l$ is the length of wings. The above results needs to be fitted to the ground coordinate system before applied to the dynamic model. In body coordinate system, the lift force of each wing acts in $X$ and $Y$ directions, and the drag force acts in $Z$ direction, so get the following moment. The Equation (9) as:

$$
\begin{aligned}
& M_{R b x}=\int_{x=R}^{x=R+1} \sum_{i=1}^{8} \sin \frac{(i-1) \pi x}{4} d U_{i}+\int_{x=R}^{x=R+1} \sum_{i=1}^{8} \sin \frac{(i+5) \pi x}{4} d m_{i} \\
& M_{R b y}=\int_{x=R}^{x=R+1} \sum_{i=1}^{8} \sin \frac{(i+1) \pi x}{4} d U_{i}+\int_{x=R}^{x=R+1} \sum_{i=1}^{8} \sin \frac{(i-1) \pi x}{4} d m_{i} \\
& M_{R b z}=\int_{x=R}^{x=R+1} \sum_{i=1}^{8} x d Q_{i}
\end{aligned}
$$

Assuming that the projection of aerodynamic force in ground coordinates does not change violently, so the CFD analysis results[1] of disc fuselage can be set as linear fitting of typical numerical calculation, expressed in form of periodic piecewise function, while two intervals is divided in the research. The Equation (10) as:

$$
\begin{aligned}
& \left.\begin{array}{c}
F_{0 d x, y, z}=a_{x, y, z} \psi^{\prime}+b_{x, y, z} \\
M_{0 d x, y, z}=c_{x, y, z} \psi^{\prime}+d_{x, y, z}
\end{array}\right\} \psi^{\prime} \in[0, \pi / 8) \\
& \left.\left.\begin{array}{rl}
F_{0 d x, y, z} & =e_{x, y, z} \psi^{\prime}+f_{x, y, z} \\
M_{0 d x, y, z} & =g_{x, y, z} \psi^{\prime}+h_{x, y, z}
\end{array}\right\} \psi^{\prime} \in[\pi / 8, \pi / 4)\right)
\end{aligned}
$$

where $a, b, \cdots h$ are fitting coefficients. In summary, the aerodynamic force in ground coordinate system and moment in body coordinate system can expressed as Equation (11)

$$
\boldsymbol{F}_{d}=\boldsymbol{F}_{R d}+\boldsymbol{F}_{0 d} \quad \boldsymbol{M}_{b}=\boldsymbol{M}_{R b}+\boldsymbol{M}_{0 b}
$$

\subsubsection{Pull of Power}

In body coordinate system, the pulling force of power devices can be expressed as Equation (12).

$$
\left[\begin{array}{c}
T_{i b x} \\
T_{i b y} \\
T_{i b z}
\end{array}\right]=\left[\begin{array}{c}
T_{i} \cos \kappa \\
T_{i} \sin \kappa \\
0
\end{array}\right]
$$

where $\kappa$ is the angle between spanwise of the wing number $i$ and the first wing. All pull force is in the plane of rotation of the aircraft, so the torque of the power device relative to the center of the disk is along the $Z$ axis in body coordinate system, which can be expressed as Equation (13). 


$$
M_{T}=\sum_{i=1}^{8} T_{i}(R+l)
$$

The representation of the pull force in ground coordinate system can be obtained by the coordinate transformation matrix.

\section{Control method}

\subsection{Dynamic Inverse Control with Robust}

Wing-disc aircraft similar to the spin satellite, gyro, spin missiles and so on, but the aircraft aerodynamic has characteristics of complex, transverse and longitudinal coupling, and serious nonlinear[2,3], the control law can be carried out with nonlinear dynamic inverse control method, and use robust dynamic inversion method to optimize[4-9]. The research aim at cruise flight, and set all the variables are observable, for multi nonlinear system, $\boldsymbol{x}$ is the state of the object parameters, $\boldsymbol{F}(\boldsymbol{x})$ and $\boldsymbol{G}(\boldsymbol{x})$ are vector and matrix of nonlinear functions, $\boldsymbol{u}$ is control. It can be expressed as Equation (14).

$$
\boldsymbol{u}=\boldsymbol{G}^{-1}(\boldsymbol{x})[-\boldsymbol{F}(\boldsymbol{x})+\boldsymbol{v}]
$$

Which $\boldsymbol{v}$ is the expected dynamics, and $\boldsymbol{v}=\boldsymbol{\omega}_{c}\left(\boldsymbol{x}_{c}-\boldsymbol{x}\right), \boldsymbol{\omega}_{c}$ is the bandwidth, $\boldsymbol{x}_{c}$ is the desired state. Due to the poor robustness of the dynamic inversion method, a integral term can be added. The Equation (15) is,

$$
\boldsymbol{v}=\boldsymbol{\omega}_{c}\left(\boldsymbol{x}_{c}-\boldsymbol{x}\right)+\boldsymbol{\omega}_{c}^{\prime} \int_{0}^{t}\left(\boldsymbol{x}_{c}-\boldsymbol{x}\right) d \tau
$$

where $\omega_{c}^{\prime}$ is bandwidth coefficient of integral controller.

\subsection{Control Strategy Analysis}

Based on the analysis of the flight mechanism, changing the pull of power can influent the rotation, and realize height control. The pull increase and decrease periodically to overcome drag during flying, and realize the speed control. Adjustment of aileron deflection angle periodically is to realize lift balance and attitude control. In addition, the aileron rudder deflection jointly can also achieve height control, which can be used as auxiliary means to achieve position control.

\subsubsection{Constraint relation of Control Quantity}

The controller consists of 8 pull power and 8 rudder, all together 16 actuators. It is necessary to design a certain allocation, to get same dimension of input of control variables and state parameters, and use the dynamic inverse control method reasonably and effectively.

Because the pull of power and rudder deflection change periodically is similar to trigonometric function, set the power device average value $T_{0}$ as a basis, the pull of two wings in the relatively position increase or decrease same value to make full use of the difference. As Equation (16).

$$
T_{i}=T_{0}+\sin \frac{(i+1) \pi}{4} \Delta T_{1}+\sin \frac{(i-1) \pi}{4} \Delta T_{3}
$$

The aileron control can make the above pulling control as a reference. As Equation (17). 


$$
\delta_{2,4,6,8}=\delta_{0}, \delta_{1}=-\delta_{5}=\Delta \delta_{1}, \delta_{3}=-\delta_{7}=\Delta \delta_{3}
$$

The control quantity reduces from 16 actuators to 6 by the constraint relation. As Equation (18).

$$
\boldsymbol{u}=\left[T_{0}, \Delta T_{1}, \Delta T_{3}, \delta_{0}, \Delta \delta_{1}, \Delta \delta_{3}\right]^{T}
$$

In body coordinate system, the control quantity has its main control direction, $T_{0}$ is for the rotation moment of the rotor, $\Delta T_{1}, \Delta T_{3}$ is for the horizontal force, $\Delta \delta_{1}, \Delta \delta_{3}$ is mainly related to the pitch and roll torque.

\subsubsection{Structure of Control}

It can be found in dynamic equation that the attitude angle $\dot{\phi}, \dot{\theta}$ and $\dot{\psi}$ is only related to $p$, $q, r$ and attitude angle in body coordinate system, so the attitude angle cannot be controlled directly, and rotating is without control. Separate inner and outer ring of control, and put roll angle $\varphi$ and pitch angle $\theta$ in the outer ring, and according to the given instructions, and make use of the relationship between dynamic attitude angle and angular velocity.

\subsection{Trim Calculation}

The control quantity in different rotated position in a given steady state of flight can be obtained by optimizing, and set derivative of speed, angular velocity and attitude angle to be 0 in body coordinate system.

Set the equilibrium flight state to a height of $18 \mathrm{~km}$, the forward flight speed $V=15 \mathrm{~m} / \mathrm{s}$, the overall elevation angle is $2^{\circ}$. Namely, in the dynamic model set $v_{x}=15 \mathrm{~m} / \mathrm{s}, v_{y}=0 \mathrm{~m} / \mathrm{s}, v_{z}$ $=0 \mathrm{~m} / \mathrm{s}, p=0 \mathrm{rad} / \mathrm{s}, q=0 \mathrm{rad} / \mathrm{s}, r=2.5133 \mathrm{rad} / \mathrm{s}$, (corresponding to $n=0.4$ revolution per second), $\varphi=0^{\circ}, \theta=2^{\circ}, \psi$ traversing from 0 to $2 \pi$. The optimal control is shown in figure 2 .
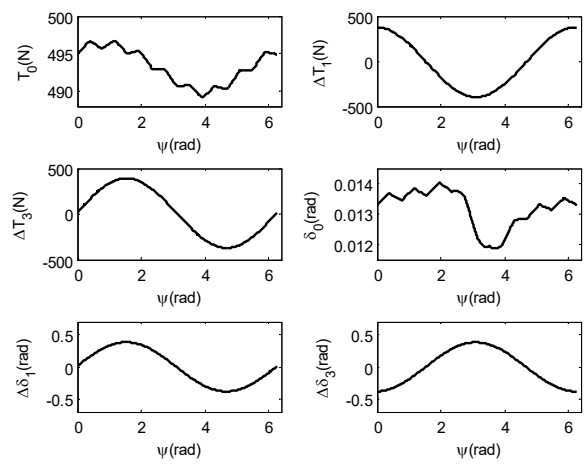

Fig. 2. Trim control curve.

\section{Design of Control}

The dynamic state quantity can be expressed as Equation (19).

$$
\boldsymbol{x}_{i n}=\left[v_{x}, v_{y}, v_{z}, p, q, r\right]^{T}
$$

In system input $\boldsymbol{u}$, force acting on the body can be converted to the ground coordinate system, rudder deflection effect can make use of the following formula, wing force can be get by further integral. As Equation (20). 


$$
\begin{aligned}
& d L_{i \delta}=\frac{1}{2} \rho W_{i}^{2} C_{l \delta} c \cdot d x \\
& d D_{i \delta}=\frac{1}{2} \rho W_{i}^{2} C_{d \delta} c \cdot d x \\
& d M_{i \delta}=\frac{1}{2} \rho W_{i}^{2} C_{m \delta} c \cdot d x \\
& d U_{i \delta}=d L_{i \delta} \cos \varphi_{0}-d D_{i \delta} \sin \varphi_{0} \\
& d Q_{i \delta}=d L_{i \delta} \sin \varphi_{0}+d D_{i \delta} \cos \varphi_{0}
\end{aligned}
$$

where $C_{l \delta}, C_{d \delta}, C_{m \delta}$ is the partial derivative of the blade aerodynamic force to the deflection angle of the rudder, $\varphi_{0}$ is the flow angle. In the design of the controller, $\boldsymbol{F}(\boldsymbol{x})$ in state equation can be obtained by setting the input $\boldsymbol{u}=0$, and as Equation (21).

$$
G(x)=G_{0} G_{1} B
$$

where $\boldsymbol{G}_{0}$ is the correlation coefficient between the mass and the moment of inertia in the dynamic model as Equation (22)

$$
\boldsymbol{G}_{0}=\operatorname{diag}\left(1 / m, 1 / m, 1 / m, 1 / I_{x}, 1 / I_{y}, 1 / I_{z}\right)
$$

$\boldsymbol{G}_{1}$ is the parameter matrix of the force and torque affected by the input of the actual controller, which can be expressed as a block matrix, Equation (23)

$$
\boldsymbol{G}_{1}=\left[\begin{array}{cc}
\boldsymbol{F}_{T} & \boldsymbol{F}_{\delta} \\
\boldsymbol{M}_{T} & \boldsymbol{M}_{\delta}
\end{array}\right]_{6 \times 16}
$$

Each of the matrix can be expressed as Equation (24-27)

$$
\begin{gathered}
\boldsymbol{F}_{T}=\boldsymbol{L}_{d b} \cdot \boldsymbol{A}_{3 \times 8} \\
\begin{array}{c}
A_{1, i}=\sin \frac{(i-1) \pi}{4}, A_{2, i}=\sin \frac{(i+1) \pi}{4}, A_{3, i}=0 \\
\boldsymbol{M}_{T}=\boldsymbol{C}_{3 \times 8} \\
C_{1, i}=0, C_{2, i}=0, C_{3, i}=R+l, \\
\boldsymbol{F}_{\delta}=\boldsymbol{L}_{d b} \boldsymbol{D}_{3 \times 8} \\
D_{1, i}=\sin \frac{(i+3) \pi}{4} \cdot Q_{i \delta}, D_{2, i}=\sin \frac{(i+5) \pi}{4} \cdot Q_{i \delta}, D_{3, i}=-U_{i \delta} \\
\boldsymbol{M}_{\delta}=\boldsymbol{E}_{3 \times 8} \\
E_{1, i}=\sin \frac{(i+5) \pi}{4} \cdot M_{i \delta}+\sin \frac{(i-1) \pi}{4} \cdot \int x d U_{i \delta} \\
E_{2, i}=\sin \frac{(i-1) \pi}{4} \cdot M_{i \delta}+\sin \frac{(i+1) \pi}{4} \cdot \int x d U_{i \delta} \\
E_{3, i}=-\int x d Q_{i \delta}
\end{array}
\end{gathered}
$$

where $\mathrm{i}=1,2, \cdots 8 . \boldsymbol{B}$ is the relationship matrix between the input and the control quantity of the actual controller, which can be expressed as Equation (28) based on the control strategy.

$$
\begin{aligned}
& \boldsymbol{B}=\left[\begin{array}{cc}
\boldsymbol{B}_{1} & 0 \\
0 & \boldsymbol{B}_{2}
\end{array}\right]_{16 \times 6} \\
& B_{i, 1}=1, B_{i, 2}=\sin \frac{(i+1) \pi}{4}, B_{i, 3}=\sin \frac{(i-1) \pi}{4}, i=1,2 \cdots 8 ; \\
& \boldsymbol{B}_{2}=\left[\begin{array}{cccccccc}
0 & 1 & 0 & 1 & 0 & 1 & 0 & 1 \\
1 & 0 & 0 & 0 & -1 & 0 & 0 & 0 \\
0 & 0 & 1 & 0 & 0 & 0 & -1 & 0
\end{array}\right]^{T}
\end{aligned}
$$


The attitude controller is designed according to the relationship between the command attitude and the corresponding angular velocity. As Equation (29)

$$
\left[\begin{array}{c}
\dot{\phi} \\
\dot{\theta}
\end{array}\right]=\left[\begin{array}{cc}
\frac{\cos \psi}{\cos \theta} & -\frac{\sin \psi}{\cos \theta} \\
\sin \psi & \cos \psi
\end{array}\right]\left[\begin{array}{l}
p \\
q
\end{array}\right]
$$

The formula is consistent with the state equation, and there is no nonlinear term, which can be used to inverse the relation directly.

\section{Simulation Verification}

According to engineering experience, the speed bandwidth is set to $0.8 \mathrm{rad} / \mathrm{s}$, the angular velocity bandwidth is set to $10 \mathrm{rad} / \mathrm{s}$, and the attitude angle bandwidth is set to $2 \mathrm{rad} / \mathrm{s}$. It is not difficult to verify that the dynamic inversion method can achieve good control. However, because the dynamic model is based on the blade element theory and CFD results, and there are simplifications and assumptions, and there is a certain gap between the given model and the real model. The wing-disc aircraft rotates to get lift, so the lift in body coordinate system is a key parameter to dynamic modeling, Increase force in $Z$ direction of body coordinate system (i.e. decrease lift provided by wings) $5 \%$ gravity. In the cruise state, the instruction of $v_{x}$ is $1 \mathrm{~m} / \mathrm{s}$ step signal after $1 \mathrm{~s}$. The simulation results are shown in figure 3.
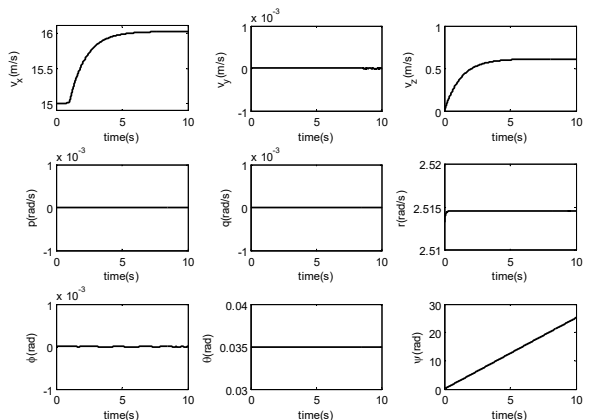

Fig. 3. Simulation of dynamic inverse control.

As can be seen from figure 3, there is static error in the velocity tracking and deviates from the trim state, The conventional dynamic inversion method cannot work well in case of model perturbation. In accordance with the robust dynamic inversion control method, the perturbation problem of the model is simulated, as shown in figure 4.
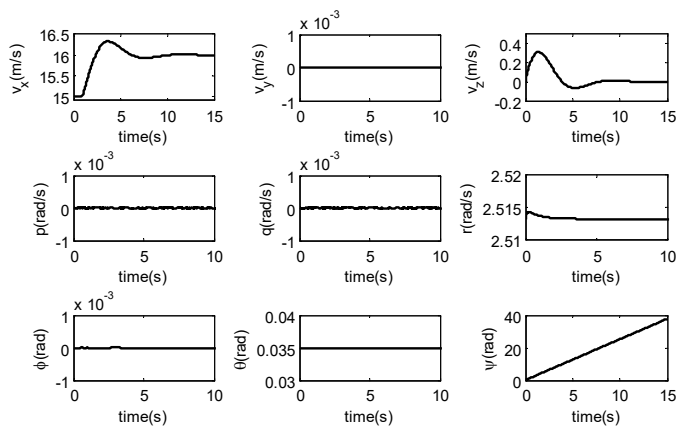

Fig. 4. Simulation of robust dynamic inverse control. 
As can be seen from figure 4, robust dynamic inversion method can achieve good speed tracking control, and other state variables are maintained at the given steady state. Robust dynamic inversion is a two order system, and overshoot appears in the tracking process, and increase the characteristic of dynamic.

\section{Conclusion}

This research focuses on the new concept of a wing-disc aircraft, establish dynamics model adaptively, and analyze the flight control method for the complicated object of control, research and proposed a new control strategy, design robust dynamic inverse control law with integral function, which is verified by simulation, laid the foundation for the detail design of the aircraft.

\section{References}

1. Hong da, Zhu jihong. Research on overall iteration design of a new concept Wing-disk Solar-powered Aircraft [C]. The 4th International Conference on Mechanical, Control and Electronic Information: (2015):6

2. Zhao Shijun, Sun Keyu, Sun Yao. Motion analysis of free rotor gyro [J]. Journal of Harbin Engineering University, (2003),03:253-257.

3. Tian Lin, $\mathrm{Xu}$ Shijie. Spin small satellite attitude dynamics modeling and control [J]. Space control technology and application, (2009),01:47-50+60.

4. Zhang Yan, Chen Zongji, Wei Chen. A robust control allocation method based on generalized inverse for UAV [J]. Journal of Aeronautics, (2008), S1:198-203.

5. ZHANG Yan, DUAN Chaoyang, ZHANG Ping(School of Automation Science and Electrical Engineering, Beijing University of Aeronautics and Astronautics). Nonlinear Autopilot Designand Simulation for Bank-to-turn Missiles Using Dynamic Inversion Method [A]. China Institute of system simulation. Proceedings of Asian Simulation Conference / The 6th International conference on system Simulation and Scientific Computing[C]. China Institute of system simulation:,(2005):5.

6. Northeastern University, IEEE electronic control system of IEEE branch, industrial association Harbin branch. The twenty-fourth China decision and Control Conference [C]. Northeastern University, IEEE electronic control system of IEEE branch, industry association Harbin branch: (2012):6.

7. XunhongLv,BinJiang,RuiyunQi,JingZhao.Survey on nonlinear reconfigurable flight control[J].Journal of Systems Engineering and Electronics,(2013),06:971-983.

8. Zou Hui, Chen Wanchun, Yin Wei. Design of feedback linearization controller for spinning missile [J]. Journal of missile and guidance, (2004), S2:228-230+234.

9. Li Nianyu, Liu Zaozhen, Xie Yongcheng. Design and Simulation of [J]. Gun Launch and control of a spinning missile attitude control system (2003), S1:30-32+38. 